\title{
Revisión bibliográfica de las recomendaciones de las Guías de Práctica Clínica para la prescripción de Medias de Compresión Médica
}

\author{
Rubén Molina Carrillo ${ }^{1}$, José María Rozas Martín ${ }^{2}$ \\ ${ }^{1}$ Enfermero. CAP Pare Claret, Instituto Catalán de la Salud \\ ${ }^{2}$ Enfermero. Hospital Universitario 12 de Octubre. Madrid \\ Correspondencia \\ Rubén Molina Carrillo \\ Correo electrónico: ruben_tmm@hotmail.com \\ Recibido: 02 de marzo de 2020 \\ Aceptado: 01 de mayo de 2020
}

\section{RESUMEN}

La terapia de compresión se puede aplicar con diversos dispositivos, entre los que se incluyen vendajes, sistemas de compresión neumática secuencial, dispositivos de velcro autoajustables, medias de compresión y los sistemas 2 en 1 compuestos por dos calcetines. Las principales indicaciones clínicas de las medias de compresión son la prevención y/o el tratamiento de los síntomas de la insuficiencia venosa primaria y el síndrome postrombótico, así como el linfedema y el lipedema. Tan solo a una minoría de los pacientes que necesitaría terapia de compresión a largo plazo se les llegan a prescribir. La inexperiencia, la falta de formación y la gran cantidad de materiales diferentes son consideradas como obstáculos por el profesional para la recomendación de medias. Por otro lado, también existe una escasa adherencia terapéutica por parte de los pacientes. El objetivo de este trabajo fue identificar y analizar las recomendaciones que ofrecen las principales guías y documentos basados en evidencias científicas para ayudar a los profesionales sanitarios a prescribir la media más adecuada para cada persona y conseguir los mejores resultados. Para ello, se han consultado 7 documentos basados en evidencias científicas. Las lagunas de conocimiento detectadas, sumadas a la confusión que se deriva de la variedad de tipos de medias disponibles, de la diversidad de condiciones clínicas que pueden requerir sus uso y de la variabilidad de situaciones personales que puede presentar cada paciente, hacen que las recomendaciones de las guías actualmente vigentes resulten insuficientes para resolver las dudas que surgen a la hora de prescribir medias de compresión.

\section{PALABRAS CLAVE}

medias de compresión; terapéutica; prescripción no medicamentosa.

\section{SUMMARY}

Compression therapy can be applied with a variety of devices, including bandages, sequential pneumatic compression systems, self-adjusting velcro devices, compression stockings, and 2-in-1 systems consisting of two socks. The main clinical indications of compression stockings are the prevention and/or treatment of symptoms of primary venous insufficiency and postrombotic syndrome, as well as lymphedema and lipedema. Only a minority of patients who would need long-term compression therapy are prescribed. Inexperience, lack of training and the large amount of different materials are considered as obstacles by the professional to the recommendation of stockings. On the other hand, there is also a low therapeutic adherence on the part of patients. The objective of this work was to identify and analyze the recommendations offered by the main guides and documents based on scientific evidence to help healthcare professionals prescribe the most appropriate stocking for each person and achieve the best results. To this end, 7 documents based on scientific evidence have been consulted. The knowledge gaps detected, coupled with the confusion that arises from the variety of types of stockings available, the diversity of clinical conditions that may require their use and the variability of personal situations that each patient may present, make the recommendations of the guidelines currently in force insufficient to resolve the doubts that arise when prescribing compression stockings.

\section{KEYWORDS}

compression stockings; therapeutics; prescriptions, non drug. 


\section{Introducción}

La compresión terapéutica supone una opción de tratamiento no invasivo y fácilmente accesible que está muy extendida y ampliamente documentada en el abordaje de enfermedades venosas y del linfedema (1). La literatura deja claro que la compresión es más eficaz que la no compresión, y que la compresión fuerte es más eficaz que la baja $(2,3,4)$.

La compresión se define como la aplicación de presión sobre un área corporal mediante un tejido elástico, o la tensión aplicada al ponerse una prenda ajustable (5). Su función principal es contrarrestar la gravedad, que es el factor clave en las perturbaciones del retorno venoso y linfático de la extremidad inferior (6) Si el nivel de compresión no compromete el flujo arterial y se emplean los medios y materiales adecuados, los efectos de la compresión pueden ser espectaculares, reduciendo el edema y el dolor y, al mismo tiempo, promoviendo la cicatrización de úlceras causadas por insuficiencia venosa (2).

La presión de compresión puede ser generada de forma externa (vendas, medias) o desde el interior, mediante la contracción de los músculos, que se expanden produciendo un aumento de la presión que realizan los vendajes. Suele expresarse en milímetros de mercurio $(\mathrm{mmHg})$ y su valor puede ser determinado por la fórmula de Laplace, mediante la siguiente ecuación: $\mathrm{P}=\mathrm{S} / \mathrm{R}$, donde $\mathrm{P}$ es la presión de compresión, $\mathrm{S}$ es la tensión del material y $\mathrm{R}$ es el radio de la extremidad (7).

La terapia de compresión se puede aplicar con diversos dispositivos, entre los que se incluyen vendajes, sistemas de compresión neumática secuencial, dispositivos de velcro autoajustables, medias de compresión médica (MCM) y los sistemas 2 en 1 compuestos por dos calcetines. Las medias de compresión tienen la ventaja de tener mayor aceptación, ser relativamente más fáciles de poner y ser menos incómodas que los vendajes y sistemas de compresión neumática (8).

Dentro de la calcetería de compresión podemos encontrar medias de soporte, medias antiembolismo y MCM (Figura 1) (8). Las medias de soporte, incluyendo calcetines de viaje y calcetines elásticos de soporte, se suelen usar para proporcionar alivio a las piernas cansadas, pesadas y dolorosas. Ejercen una presión uniforme y menor que las MCM, no están obligadas a cumplir las estrictas especificaciones técnicas que se les exige a las graduadas y pueden ser adquiridas sin receta en tiendas (8).

Las medias antiembolismo están diseñadas para pacientes encamados y se usan para reducir el riesgo de trombosis venosa profunda (TVP). Al igual que las MCM, ejercen gradiente de presión, y a menudo ambos términos se emplean indistintamente, pero ofrecen distintos niveles de compresión y tienen diferentes indicaciones (8).

\begin{tabular}{|c|c|c|c|}
\hline & Graduadas o Médicas & Antiembolismo & No médicas \\
\hline $\begin{array}{l}\text { Indicación } \\
\text { patología }\end{array}$ & EVC y el edema & Prevención de TVP & $\begin{array}{c}\text { Medias de apoyo no médicas, incluyendo } \\
\text { calcetines de vuelo y medias elásticas de soporte, se } \\
\text { usan a menudo para aliviar las piernas cansadas, } \\
\text { pesadas y dolorida }\end{array}$ \\
\hline $\begin{array}{l}\text { Indicación de } \\
\text { diseño }\end{array}$ & $\begin{array}{l}\text { Pacientes } \\
\text { ambulatorios }\end{array}$ & $\begin{array}{l}\text { Pacientes } \\
\text { encamados }\end{array}$ & $\begin{array}{l}\text { No necesitan cumplir con las especificaciones } \\
\text { médicas y técnicas estrictas }\end{array}$ \\
\hline Normativa & $\begin{array}{c}\text { Estrictas especificaciones } \\
\text { médicas y técnicas (consistencia, } \\
\text { presión en tobillo y la graduación de } \\
\text { la compresión...) }\end{array}$ & $\begin{array}{l}\text { no cumplen con las } \\
\text { especificaciones técnicas } \\
\text { para uso de pacientes } \\
\text { ambulatorios }\end{array}$ & $\begin{array}{l}\text { Ejercen una compresión considerablemente menor } \\
\text { que las medias de compresión graduadas } \\
\text { NO CERTIFICADA }\end{array}$ \\
\hline $\begin{array}{l}\text { Gradiente de } \\
\text { presión }\end{array}$ & $\begin{array}{c}\text { Mayor grado de compresión en el } \\
\text { tobillo,y el nivel de } \\
\text { compresión disminuye } \\
\text { gradualmente }\end{array}$ & $\begin{array}{l}\text { Proporcionan compresión } \\
\text { gradiente }\end{array}$ & Compresión es uniforme y no graduada \\
\hline
\end{tabular}

Figura 1. Diferencias entre compresión médica y compresión no médica

Las MCM suelen emplearse para tratar la enfermedad venosa crónica y el edema. Están diseñadas para pacientes ambulatorios y se fabrican bajo estrictas exigencias médicas y técnicas (8). El gradiente de presión asegura el retorno de la sangre hacia el corazón, evitando el reflujo hacia el pie o hacia el sistema venoso superficial. La compresión graduada puede revertir la hipertensión venosa, potenciar la acción de la bomba musculoesquelética, facilitar el retorno venoso y mejorar el drenaje linfático (9). 
El grado o nivel de compresión es la presión ejerci$\mathrm{da}$, expresada en $\mathrm{mm} \mathrm{Hg}$, a nivel del tobillo, y esta cifra es la que aparece reflejada en los envoltorios de las prendas. Esta cifra es uno de los factores que determinan su eficacia hemodinámica, y se incorpora a las MCM a través del tipo de hilos y de las técnicas textiles empleados en su fabricación.
Según la presión ejercida, hay diferentes clases de medias disponibles (6). La presión ejercida mediante cada clase varía dependiendo del país (Figura 2) y son determinados por diferentes métodos de laboratorio $(2,9)$.

\begin{tabular}{|l|c|c|c|}
\cline { 2 - 4 } \multicolumn{1}{c|}{} & $\begin{array}{c}\text { Norma britanica } \\
\text { BS 6612:1985 }\end{array}$ & $\begin{array}{c}\text { Norma francesa } \\
\text { ASQUAL }\end{array}$ & $\begin{array}{c}\text { Norma alemana } \\
\text { RAL-GZ 387:2000 }\end{array}$ \\
\hline Metodo de evaluación & HATRA & IFTH & HOSY \\
\hline Clase I & $14-17 \mathrm{mmHg}$ & $10-15 \mathrm{mmHg}$ & $18-21 \mathrm{mmHg}$ \\
\hline Clase II & $18-24 \mathrm{mmHg}$ & $15-20 \mathrm{mmHg}$ & $23-32 \mathrm{mmHg}$ \\
\hline Clase III & $25-35 \mathrm{mmHg}$ & $20-36 \mathrm{mmHg}$ & $34-46 \mathrm{mmHg}$ \\
\hline Clase IV & No descrita & $>36 \mathrm{mmHg}$ & $>49 \mathrm{mmHg}$ \\
\hline $\begin{array}{l}\text { Los rangos en mmHg corresponden a la presión aplicada en la mínima circunferencia en tobillo } \\
\text { (punto B en la escala internacional de compresión graduada) }\end{array}$
\end{tabular}

Figura 2: Normativas europeas de medias de compresión. Fuente: GPC de la AEEVH (10)

Muchas marcas de medias siguen la norma alemana, y aplican gradiente de presión a sus prendas. La presión que ejercen a nivel de la pantorrilla (nivel C) oscila entre el 50 y el $80 \%$ de la presión del tobillo (nivel B), y la que ejercen a nivel del muslo (nivel D) está entre el 20 y el $60 \%$ de la presión del tobillo. Según el Comité Europeo de Normalización (CEN), la presión a los niveles $C$ y $D$ debe ser del $80 \%$ y del $50 \%$ de la presión ejercida a nivel $\mathrm{B}(11)$.

La rigidez de las prendas resulta también crucial para predecir la efectividad de las prendas y la tolerancia de los pacientes (12). Según el CEN, la rigidez se define como el aumento que experimenta la presión que ejerce la media por cada cm que aumente el perímetro de la pierna a la altura del tobillo, y se expresa en $\mathrm{mmHg}$. Cuanto mayor es la rigidez de una prenda de compresión mayor es la mejoría obtenida en los parámetros hemodinámicos y la eficacia para reducir el edema (1). Sin embargo, no existen estándares para medir la rigidez, no se comprueba de forma rutinaria, ni se indica en las etiquetas o embalajes de las prendas (5).

La presión de reposo es la presión ejercida sobre la pierna cuando el paciente está en decúbito supino y con las articulaciones de tobillo y rodilla relajadas (13). El índice de rigidez estática es la diferencia que existe entre la presión que la prenda ejerce cuando el paciente está de pie y la presión de reposo (13). La actividad muscular produce importantes cambios en el perímetro de la pierna, lo que produce un aumento en la presión que ejercen los vendajes. El índice de rigidez dinámica mide la diferencia entre los picos de presión que se producen al caminar (presión de trabajo) y la presión de reposo (14).
La fabricación de las MCM puede ser estándar o a medida (6). La confección se puede realizar con tricotado circular o plano y se encuentran en diferentes tallas y modelos, desde el calcetín hasta medias con extensión a cadera o panty completo con protección de dedos según necesidades (10).

Las medias de tricotado circular se tejen en círculos, sin costuras, y se fabrican por tallas. Suelen estar indicadas para edemas poco voluminosos (19). En cambio, las medias de tricotado plano se tejen como una sola pieza que se une por una costura, así pues, deben ser realizadas a medida (19). De esta manera se consigue un tejido más rígido, lo que lo hace más indicado para edemas grandes (19).

Las principales indicaciones clínicas de las MCM son la prevención y/o el tratamiento de los síntomas del linfedema, lipedema y la enfermedad venosa crónica (tanto la insuficiencia venosa primaria como el síndrome postrombótico). Las medias también se emplean en el tratamiento de edemas de otras causas como embarazo, inmovilidad, insuficiencia cardiaca, insuficiencia renal, obesidad o ciertos fármacos $(5,15)$. Por su efecto antiinflamatorio, la compresión también se recomienda en caso de celulitis y ciertas vasculitis (15).

El uso de medias de compresión no está exento de riesgos. Entre sus contraindicaciones podemos encontrar: presencia o sospecha de enfermedad arterial periférica (incluyendo antecedentes de revascularización), neuropatía severa, alergia a los materiales de las medias, edema pulmonar por insuficiencia cardiaca, afecciones dermatológicas o de partes blandas (incluyendo injertos cutáneos recientes, dermatoporosis, gangrena, dermatitis exudativa y celulitis grave) y deformidades o tallas extremas que impidan una adaptación adecuada 
de las prendas. Unas medias mal ajustadas pueden generar disconfort y, en el peor de los casos, necrosis por presión. En pacientes con insuficiencia arterial pueden empeorar la isquemia, y los pacientes alérgicos pueden desarrollar dermatitis de contacto (8).

Es sorprendente que, contrariamente a lo que recomiendan las Guías de Práctica Clínica (GPC), tan solo a una minoría de los pacientes que necesitaría terapia de compresión a largo plazo se les llegan a prescribir (18). La inexperiencia, la falta de formación y la gran cantidad de materiales diferentes son considerados como obstáculos por el profesional para la recomendación de medias terapéuticas $(4,20)$.

Por otro lado, también existe una escasa adherencia terapéutica por parte de los pacientes; dependiendo de los estudios, el incumplimiento oscila entre el $10 \mathrm{y}$ el $80 \%(17,18)$. Entre los motivos de la falta de adherencia se encontraba el disconfort por calor o irritación de la piel, incomodidad, corte de circulación, el dolor en las extremidades, costes, apariencia y la falta de ayudas para su colocación (17).

Con demasiada frecuencia, elecciones inadecuadas dejan a los pacientes frustrados, desesperados o abatidos, y tienen un impacto negativo sobre el éxito en el control del edema a largo plazo.

El objetivo de este trabajo fue identificar y analizar las recomendaciones que ofrecen las principales GPC y otros documentos basados en evidencias científicas para ayudar a los profesionales sanitarios a seleccionar la MCM más adecuada para cada persona y conseguir los mejores resultados. Como objetivos específicos, identificar las principales indicaciones de las medias de compresión, las recomendaciones existentes sobre el nivel de compresión que se debe aplicar en cada caso, saber cómo elegir el tipo de prenda más adecuado para cada paciente y descubrir qué pautas se ofrecen para mejorar la eficacia de las medias y la adherencia terapéutica de los pacientes.

\section{Metodología}

Para realizar este trabajo se llevó a cabo una búsqueda de artículos y documentos en las páginas web de sociedades científicas, portales de salud, organismos elaboradores y/o repositores de GPC, bases de datos y metabuscadores, entre abril y mayo de 2020.

La búsqueda de GPC y documentos de consenso basados en evidencias se realizó en GuiaSalud, Fisterra, National Institute for Clinical Excellence (NICE), National Health and Medical Research Council (NHMRC), Canadian Medical Association (CMA INFOBASE), Registered Nurses' Association of Ontario (RNAO), Scottish Intercollegiate Guidelines Network (SING), Asociación Española de Enfermería Vascular y Heridas (AEEVH), Grupo Nacional para el Estudio y Asesoramiento en Úlcera Por Presión y Heridas Crónicas (GNEAUPP), Sociedad Española de Angiología Y Cirugía Vascular (SE$A C V)$, European Society for Vascular Surgery (ESVS),
European Wound Management Association (EWMA) y Medline.

Los criterios de inclusión de Guías y Documentos de Consenso fueron: estar vigentes, ser de acceso libre y estar disponibles en español o inglés.

Los duplicados y documentos que no cumplían criterios de inclusión fueron desestimados. Solo se realizó una lectura completa de los artículos que cumplían criterios de inclusión. Los documentos que no incluían recomendaciones o consejos para la prescripción de MCM o para mejorar la adherencia terapéutica también fueron desestimados. Aunque no se realizó una evaluación de la calidad, se dio prioridad a aquellas guías y documentos de consenso que basaban sus recomendaciones en Revisiones Sistemáticas (RS) de la literatura. Finalmente, todo este proceso se complementó con una búsqueda manual en las listas de referencias de los documentos hallados.

\section{Resultados}

Para realizar esta revisión se han consultado 7 documentos basados en evidencias científicas $(1,21,22,23$, $24,25,26)$ Cuatro de estos documentos fueron elaborados en España por un Portal de Salud (22) y por tres sociedades científicas $(23,24,26)$. El resto fueron elaborados por dos sociedades científicas europeas $(1,25)$ y un grupo de expertos de ocho países europeos (21). Tan solo dos de los documentos $(1,21)$ incorporaron el sistema GRADE para clasificar la fuerza de sus recomendaciones.

1. Circunstancias en las que se recomienda el uso de MCM

- En el tratamiento de síntomas y complicaciones de la insuficiencia venosa $(21,25)$.

- En la prevención del edema en pacientes con insuficiencia venosa o pacientes sanos con riesgo de edema (ej.: vuelos y edema ocupacional) (21).

- En la prevención de recidivas de úlceras venosas (21).

- En pacientes con lipedema solo se recomienda el uso de MCM si existe edema asociado (24).

- En la fase inicial del posoperatorio de varices: safenectomías, ablaciones endovenosas y escleroterapia. $(1,21,23)$.

- No se recomienda, de forma sistemática, el uso prolongado de MCM en el posoperatorio de safenectomías, salvo en aquellos pacientes con síntomas de insuficiencia venosa crónica (21).

- No hay suficiente evidencia para recomendar el uso de MCM para evitar la progresión de la enfermedad venosa crónica (21).

- En Trombosis Venosa Profunda (TVP), para reducir el edema y el dolor y para evitar la propagación del trombo (21). 
- En trombosis venosas superficiales (21).

- En la profilaxis del Síndrome Postromótico (SPT) tras TVP (21).

- En SPT sintomáticos (21).

- En pacientes que van a ser sometidos a cirugía mayor se recomienda el uso de medias antiembolismo para prevenir TVP (21).

- En vuelos de larga distancia, para prevenir TVP (21).

- En el tratamiento de mantenimiento del linfedema $(21,26)$.

2. Nivel de compresión que se debe aplicar, precauciones y contraindicaciones

- Se recomienda el uso de medias de clase 1 (normativa alemana) en los estadios CO y C1 de la CEAP (piernas cansadas, telangiectasias,varices de pequeño tamaño o asintomáticas), varicosis incipiente del embarazo, prevención del edema ortostático y prevención del linfedema (estadio $0 / I)(22,24,26)$.

- Se recomienda el uso de medias de clase 2 ( normativa alemana) en insuficiencia venosa moderada con varices tronculares $(22,24)$, y también en la postcirugía de varices, post-escleroterapia, prevención de la TVP, linfedema estadio I-II, post-liposucción $(24,26)$.

- Se recomienda el uso de medias clase 2 (Normativa alemana) en los estadios C2 y C3 de la CEAP (22).

- Se recomienda el uso de medias de clase 3 (normativa alemana) en insuficiencia venosa severa con cambios tróficos cutáneos, edema post-trombótico y linfedema estadio II/III en MMII $(24,26)$.

- Se recomienda el uso de medias de clase 3 ( normativa alemana) en los estadios C4 y C5 de la CEAP (22).

- Se recomienda el uso de medias de clase 4 ( normativa alemana) en úlceras venosas y linfedema severo $(24,26)$.

- Las medias de clase 3 (normativa alemana) reducen la incidencia de SPT (23).

- En el posoperatorio de varices las medias de clase 1 son tan eficaces como las de clase 3 (23).

- En pacientes con úlcera venosas se recomienda emplear compresión fuerte (mayor o igual a 40 $\mathrm{mmHg})(1,25)$.

- Las presiones por encima de $49 \mathrm{mmHg}$ se usan en linfedemas severos complejos de miembros inferiores (estadio III) y pueden llegar hasta $70 \mathrm{mmHg}$ siempre que no exista enfermedad arterial acompañante.

- En pacientes con úlceras venosas y compromiso arterial, si el ITB es mayor de 0,5 o la TA sistólica a nivel del tobillo es superior a $60 \mathrm{mmHg}$, se puede emplear compresión reducida $(25,27)$.

- En pacientes con ITB menor de 0,5 no se debe aplicar compresión (27).
- En pacientes con ITB mayor de 1,3 realizar palpación de pulsos y valorar las ondas del doppler. Derivar a cirugía vascular o centro especializado si es necesario (27).

\section{Tipo de media que se debe prescribir}

- La mayoría de los pacientes con insuficiencia venosa responderán bien al uso de medias cortas, pero en aquellos que presenten varices sintomáticas en el muslo está indicado el uso de medias altas (22).

- En pacientes que han sufrido ictus se recomienda el uso de medias por encima de la rodilla para prevenir TVP.

- En el tratamiento de úlceras venosas se recomienda el uso de kits de calcetines (25).

- Las alteraciones importantes de la forma del miembro se benefician más del tejido plano (26).

- Las prendas prefabricadas están indicadas en la insuficiencia venosa (26)

- Las prendas a medida están indicadas en el linfedema, especialmente las de clase 3 y 4 (26.)

- En pacientes con linfedema del miembro inferior, los calcetines elásticos abiertos pueden aumentar el edema de los dedos, por ello es recomendable prescribir el guantelete de los dedos (26).

4. Recomendaciones para mejorar la efectividad y la adherencia terapéutica

- Es recomendable realizar educación sanitaria sobre la enfermedad, los beneficios de la terapia y sobre cómo colocarse y retirarse las medias.

- La toma de medidas se realizará cuando el edema haya sido minimizado con terapia descongestiva $(26,27)$.

- Si el paciente no aguanta la presión que necesita se prescribirán presiones inferiores (26).

- El recambio de la prenda se realizará cada 6 meses o cuando pierda sus propiedades de elasticidad. EI tejido plano es más duradero, a veces pueden mantener su eficacia hasta un año (26).

- El tejido circular tiene una cosmética más aceptable y es más fácil de colocar (26).

\section{Discusión}

A pesar de que hay suficientes evidencias científicas que respaldan el uso de medias de compresión en diversas situaciones, son pocas las GPC que ofrecen recomendaciones que puedan resultar de utilidad a la hora de prescribir estas prendas. La mayor parte de documentos consultados se limitan a destacar la eficacia de la terapia de compresión para evitar y/o tratar síntomas y complicaciones asociados a los trastornos venosos y linfáticos, pero hacen pocas recomendaciones concretas que, en la práctica diaria, puedan servir 
de ayuda a los profesionales a la hora de saber qué propiedades deben reunir las prenda dependiendo del problema de base, la situación clínica y otras características del paciente.

La mayoría de las recomendaciones halladas están relacionadas con las diferentes situaciones en las que se debe usar MCM y con la presión que estas deben ejercer, pero hay muy pocas indicaciones relativas al tipo de tejido, la longitud necesaria o la forma de mejorar la adherencia terapéutica.

El uso de medias de compresión está aconsejado en casi todas las situaciones donde puede haber edema, pero no se han encontrado recomendaciones sobre su uso en pacientes con edemas de origen sistémico ni en pacientes con edema por revascularización. Con respecto al edema por revascularización, el documento de consenso basado en evidencias (21) sugiere que el uso de MCM ligeras (15-21 mmHg) podría ser una medida eficaz, pero que de momento no se dispone de suficiente evidencia como para poder recomendarlas. Con relación al edema secundario a insuficiencia cardiaca congestiva grave, ese mismo documento (21) contraindica las MCM, pero sugiere que si el edema no es importante se pueden emplear MCM ligeras. Respecto a otras situaciones donde podría estar indicado el uso de MCM como el edema secundario a embarazo o al uso de fármacos y procesos inflamatorios que se acompañan de edema (como celulitis, vasculitis y patología traumatológica y del deporte) tampoco se han encontrado recomendaciones.

A pesar de que algún documento recomienda el uso de MCM para la prevención y tratamiento del SPT secundario a TVP, una RS Cochrane publicada en 2019 (28) cuestiona estas recomendaciones al concluir que falta evidencia para poder apoyar el uso de compresión en la prevención del SPT y que se necesitan estudios para determinar si la compresión puede aliviar los síntomas producidos por el SPT.

Aunque hay tres tipos de normativas que regulan el nivel de compresión y cada marca comercial utiliza una en el etiquetado, la mayor parte de las guías utilizan la alemana (RAL-GZ 387), pero es lógico pensar que los profesionales deberían estar familiarizados con las tres normativas para poder escoger entre los diferentes modelos que ofertan todas las marcas comerciales. Para complicar más las cosas, hay documentos donde, en vez de utilizar alguna de las tres normativas, se hace referencia a compresión ligera, normal y fuerte.

Se ha hallado unanimidad en el hecho de considerar la isquemia crítica como una contraindicación para el uso de MCM. Por otro lado, en el caso de que haya enfermedad arterial leve subyacente (IT/B $>0,5$ ) solo se sabe que se deben usar presiones reducidas, pero ninguna GPC recomienda una clase de media concreta.
Tampoco se han encontrado recomendaciones dirigidas a la población diabética, tan solo se recomienda que, ante la sospecha de calcificación de los troncos tibio-peroneos (IT/B>1,3) se deben tomar precauciones.

La práctica más habitual suele consistir en prescribir las medias que están financiadas por el sistema sanitario. En España se suelen recetar las de compresión fuerte (categorización que, como ya se ha comentado, no se corresponde con ninguna de las normas europeas) y en tricotado circular (las de tricotado plano requieren autorización por parte de la Inspección) y se remite al paciente a la farmacia para su adquisición, pero la lectura de la literatura disponible induce a pensar que, para que la prescripción sea realmente eficaz, se requeriría un proceso de selección mucho más complejo en el que debería estar implicado el propio paciente y que permitiese tener en cuenta aspectos tan variados como su habilidad, su movilidad y su situación económica. Aparte de realizar una adecuada valoración clínica inicial que permita detectar contraindicaciones, es necesario determinar qué nivel de compresión se necesita, qué tipo de tejido, qué longitud de media y valorar si se necesita una prenda confeccionada a medida o no. Una vez tomadas esas decisiones, se debería tener en cuenta que, si el paciente presenta edema, habría que reducirlo mediante vendajes o sistemas de velcro antes de la toma de medidas. Con respecto a este último asunto, lo ideal sería remitir al paciente a una ortopedia certificada.

En algunos documentos seleccionados se han encontrado recomendaciones contradictorias sobre cuál es el nivel de compresión óptimo que requieren ciertas situaciones $(21,22,23)$. Probablemente esto sea debido a que haya variabilidad metodológica en los estudios analizados, pero también podría deberse a la falta de consenso sobre la normativa a seguir a la hora de recomendar la clase de compresión requerida (24).

Según algún autor, potenciar la adherencia es más importante que la selección del nivel de compresión (5). Por lo tanto, la prescripción de medias debe estar basado en un enfoque holístico e individualizado que valore tanto las capacidades físicas, como psicológicas y culturales de la persona (6). Acciones como tomar medidas, ajustar la presión al máximo tolerable, priorizar el uso de tejido circular y realizar educación sanitaria son algunas de las recomendaciones halladas, pero según el documento STRIDE (5) hay otras opciones que podrían ser muy útiles, como por ejemplo la superposición de medias de las mismas o de diferentes características (a imitación de lo que se consigue con los kit de úlceras compuestos por dos calcetines), que es una forma de aumentar la rigidez al mismo tiempo que se facilita la aplicación y retirada de las medias. Además, 
esto protege las pieles frágiles y permite el uso de apósitos debajo de las medias.

Hay edemas que aumentan solo durante el día y se resuelven durante el descanso, y otros que aumentan durante el día y la noche. Según el documento STRIDE (5), hay circunstancias en las que debería recomendarse el uso nocturno de compresión, pero en las GPC consultadas tampoco se ha hallado ninguna recomendación al respecto.

\section{Conclusión}

Las lagunas de conocimiento detectadas, sumadas a la confusión que se deriva de la gran variedad de medias disponibles, de la diversidad de condiciones clínicas en las que puede estar aconsejado su uso, y de la variabilidad de situaciones personales que puede presentar cada paciente, hacen que las recomendaciones de las GPC resulten insuficientes para resolver las dudas que surgen a la hora de prescribir medias de compresión.

La isquemia crítica es una contraindicación absoluta. Cuando el paciente no tolere el nivel de compresión que necesita se prescribirán presiones inferiores.

Para poder prescribir con garantías el tipo de prenda más adecuado para cada paciente (o poder elaborar algún tipo de protocolo o algoritmo) sigue siendo necesario recurrir a evidencias de menor calidad como los consejos de los expertos y tener en cuenta tanto la experiencia del profesional como las preferencias de los pacientes.

\section{Consideraciones éticas}

Los autores de esta revisión declaran ausencia de conflictos de interés que puedan haber influido en su realización. Ambos autores han participado tanto en la localización de documentos como en la extracción y discusión de los resultados.

\section{Bibliografía}

1. Wittens C, Davies AH, Baekgaard N, Broholm R, Cavezzi A, Chastanet S, et al. Editor's choiceManagement of chronic venous disease. Clinical Practice Guidelines of The European Society for Vascular Surgery (ESVS) [Internet]. Eur J Vasc Endovas Surg. 2015; 49 (6): 678-37 [cited 2020 May 15]. Disponible en: https://doi.org/10.1016/j.ejvs.2015.02.007

2. European Wound Management Association. Comprendiendo la terapia compresiva [Internet]. Medical Education Partnership LTD, editor. Oxford; 2003. Disponible en:

https://www.aeev.net/guias/Spring_2003_Spanish -.pdf
3. O'Meara S, Cullum N, Nelson EA, Dumville JC. Compression for venous leg ulcers. Cochrane Database Syst Rev. 2012; 14;11(11):CD000265. doi: 10.1002/ 14651858.CD000265.pub3.

4. Nelson EA, Bell-Syer SE. Compression for preventing recurrence of venous ulcers. Cochrane Database Syst Rev. 2012;(8):CD002303. doi:10.1002/14651858. CD002303.pub2

5. Bjork R, Ehmann S. S.T.R.I.D.E. Professional Guide to Compression Garment Selection for the Lower Extremity. J Wound Care. 2019;28(Sup6a):1-44. doi:10. 12968/jowc.2019.28.Sup6a.S1

6. Partsch H. Compression therapy: clinical and experimental evidence. Ann Vasc Dis. 2012;5(4):416422. doi:10.3400/avd.ra.12.00068

7. Thomas $\mathrm{S}$. The use of the Laplace equation in the calculation of sub-bandage pressure. EWMA journal. 2003; 3 (1): 21-23.

8. Chung SL, Alun HD. Graduated compression stockings. CMAJ.2014; 186(10): 391-8. doi: 10.1504/cmaj. 131281

9. Moffatt C. Variability of pressure provided by sustained compression. Int Wound J. 2008;5(2):259-65. doi:10.1111/j.1742-481X.2008.00470.x

10. Asociación Española de Enfermería Vascular y Heridas. Guía de Práctica Clínica: Consenso sobre úlceras vasculares y pie diabético. Tercera edición. Madrid: AEEVH, 2017.[cited 2020 Feb 29]. Disponible en: https://www.aeev.net/pdf/Guia-de-Practica-Clinicaweb.pdf

11. European Committee for standardization (CEN). Non-active medical devices. Working group 2 ENV 12718: European pre-standard "Medical Compression Hosiery". CEN TC205. Brussels: CEN; 2001.

12. Blättler W, Zimmet SE. Compression therapy in venous disease. Phlebology. 2008;23(5):203-205. doi: 10.1258/phleb.2008.081004

13. Partsch H, Schuren J, Mosti G, Benigni JP. The Static Stiffness Index: an important parameter to characterise compression therapy in vivo. J Wound Care. 2016;25 Suppl 9:S4-S10. doi:10.12968/jowc.2016. 25.Sup9.S4

14. Kumar B, Das A, Alagirusamy R. Analysis of subbandage pressure of compression bandages during exercise. J Tissue Viability. 2012;21(4):115-24. doi: 10.1016/j.jtv.2012.09.002

15. Rabe E, Partsch H, Hafner J, Lattimer C, Mosti G, Neumann $M$, et al. Indications for medical compression stockings in venous and lymphatic disorders: An evidence-based consensus statement [Internet]. Vol. 33, Phlebology. SAGE Publications Ltd; 2018 [cited 2020 May 22]. p. 163-84. Disponible en: https://www.ncbi.nlm.nih.gov/pmc/articles/PMC58 46867/ 
16. Eklof B, Rutherford RB, Bergan JJ, Carpentier PH, Glovizki P, Kistner RL et al. Revision of the CEAP classification for chronic venous disorders: consensus statement. J Vasc Surg. 2004; 40: 1248-52. doi: 10.1016/j.jvs.2004.09.027

17. Raju S, Hollis K, Neglen P. Use of Compression Stockings in Chronic Venous Disease: Patient Compliance and Efficacy. Ann Vasc Surg. 2007; 21 (6): 790-5. doi:10.1016/j.avsg.2007.07.014

18. Rabe E, Hertel S, Bock E, Hoffmann B, Jöckel KH, Pannier F. Therapy with compression stockings in Germany - results from the Bonn Vein Studies. J Dtsch Dermatol Ges. 2013;11(3):257-61. doi: 10.1111/j.1610-0387.2012.08048.x

19. Ritchie G, Freeman N. Understanding compression: Part 3-compression hosiery stockings and adjustable wraps. Journal of Community Nursing. 2018; 32 (4):20-8.

20. Raña-Lama CD, Bouza-Prego MA, Saleta-Canosa JL,Rumbo-Prieto JM, Arantón-Areosa L. Obstáculos y apoyos percibidos en la aplicación de terapia compresiva en úlceras venosas de la pierna. Ene [Internet]. 2016; 10(2). Disponible en:

http://scielo.isciii.es/scielo.php?pid=S1988348X2016000200004\&script=sci_arttext\&tlng=pt

21. Rabe E, Partsch $H$, Hafner J, et al. Indications for medical compression stockings in venous and lymphatic disorders: An evidence-based consensus statement. Phlebology. 2018;33(3):163-184. doi: $10.1177 / 0268355516689631$

22. Villa Estebanez R, Veiras del Río O, De la Fuente Laso P. Guía Clínica de Insuficiencia Venosa Crónica. [Internet]. Madrid: Fisterra. 2018 [cited 2020 May 16]. Disponible en: https://www-fisterra-com.m-hdoct.a17.csinet.es/ guias-clinicas/insuficiencia-venosa-cronica/
23. Miquel Abbad C, Rial Horcajo R, Ballesteros Ortega MD, García Madrid C. Practice guidelines in chronic venous disease from the Phlebology and Lymphology Working Group of the Spanish Society of Angiology and Vascular Surgery. Angiologia [Internet]. 2016 [cited 2020 May 22];68(1):55-62. Available from: https://www.elsevier.es/es-revista-angiologia294-articulo-guia-practica-clinica-enfermedadvenosa-S0003317015002084

24. Alcolea J, Alfonso B, Arroyo A, Domingo P, Galindo A, Gracia $M$, et al. Documento de Consenso Lipedema [Internet]. Madrid: Asociación Española de Linfedema y Lipedema; 2018. Available from: https://aelinfedema.org/wp-content/uploads/2019/ 11/Consenso-Lipedema-v.Sep-2019.pdf

25. Franks PJ, Barker J, Collier M, et al. Management of Patients With Venous Leg Ulcers: Challenges and Current Best Practice. J Wound Care. 2016;25 Suppl 6:S1-S67. doi:10.12968/jowc.2016.25.Sup6.S1

26. Puigdellivol Serafí C, Alonso Álvarez B. Orientación Diagnóstica y Terapéutica del Linfedema [Internet]. Madrid: Capítulo Español de Flebología y Linfología; 2017 [cited 2020 May 22]. Available from: https://www.mision-compresion.es/upload/ publicaciones/PDF3_Guia-linfedema-segundaedicion-2017_439.pdf

27. Chitambira F. Patient perspectives: explaining low rates of compliance to compression therapy. Wound Pract Res. 2019;27(4).

28. Azirar S, Appelen D, Prins MH, Neumann MH, de Feiter AN, Kolbach DN. Compression therapy for treating post-thrombotic syndrome. Cochrane Database Syst Rev [Internet]. 2019 Sep 18;(9). Disponible en: https://www.cochranelibrary.com/es/cdsr/doi/10.1 002/14651858.CD004177.pub2/full/es 